\title{
RIGIDITY OF SOME TRANSLATIONS ON HOMOGENEOUS SPACES
}

\author{
BY DAVE WITTE
}

Ornstein and Weiss $[\mathbf{2}]$ proved that the geodesic flow on a compact surface of constant negative curvature manifests extreme randomness (it is Bernoulli). In contrast, Ratner [3] has shown that the horocycle flow is very rigid: a measure-theoretic isomorphism between two horocycle flows must be an affine map (a.e.). More concretely, suppose $\Gamma$ and $\Lambda$ are lattices in $G=\mathrm{SL}_{2}(\mathbf{R})$, and let $u=\left(\begin{array}{ll}1 & t \\ 0 & 1\end{array}\right)$ be a (nonidentity) unipotent element of $G$. Ratner showed that if $\psi: G / \Gamma \rightarrow G / \Lambda$ is a $u$-equivariant measure-preserving Borel map, then $\psi$ is an affine map (a.e.). Here we announce the proof of a satisfactory extension of this rigidity theorem to the situation where $G$ is replaced by any connected semisimple Lie group.

A discrete subgroup $\Gamma$ of $G$ is a lattice if the homogeneous space $G / \Gamma$ has finite volume. We say $\psi: G / \Gamma \rightarrow H / \Lambda$ is affine for $g \in G$ if there is some $h \in H$ with $\psi(\Gamma x g)=\psi(\Gamma x) \cdot h$ for a.e. $\Gamma x \in G / \Gamma$. Obviously, $\psi$ is affine for $g$ if $\psi$ is affine for $G$ : i.e., if $\psi$ is affine for each element of $G$. The subject of this announcement is a converse to this statement-for translations of zero entropy (this includes the unipotent translations). Note that if $H$ acts faithfully on $H / \Lambda$, then any measure-preserving map $\psi: G / \Gamma \rightarrow H / \Lambda$ that is affine for $G$ is an affine map (a.e.); i.e., there is a continuous surjective homomorphism $\sigma: G \rightarrow H$ and some $h_{0} \in H$ such that $\psi(\Gamma x)=\Lambda h_{0} \cdot \sigma(x)$ for a.e. $\Gamma x \in G / \Gamma$.

THEOREM. Suppose $\Gamma$ and $\Lambda$ are lattices in connected semisimple Lie groups $G$ and $H$. If $\psi: G / \Gamma \rightarrow H / \Lambda$ is a measure-preserving Borel map that is affine for a zero entropy ergodic translation of $G / \Gamma$, then $\psi$ is affine for $G$.

It is easy to prove the theorem under a hypothesis of high $\mathbf{R}$-rank. We begin by showing that if $\psi$ is affine for a zero entropy ergodic translation $g$, then $\psi$ is affine for every element of the connected centralizer $C_{G}(g)^{0}$. (This is based on polynomial divergence of orbits - the argument is due to Ratner.) Repeating the argument, we know $\psi$ is affine for centralizers of ergodic unipotent elements of $C_{G}(g)^{0}$, and for centralizers of unipotent elements of these centralizers, and so on. In high $\mathbf{R}$-rank we eventually reach a collection of centralizers that generate $G$-thus $\psi$ is affine for $G$. The case of low $\mathbf{R}$-rank takes more work. Because the centralizers do not generate, we need to base arguments on commutation relations satisfied by elements of subgroups of $G$ (cf. the proof of Lemma 3.4 in [3]).

When $G$ and $H$ are connected noncompact simple Lie groups with finite center, we can construct ergodic $G$-actions by embedding $G$ in $H$ : then $G$ acts

Received by the editors April 23, 1984.

1980 Mathematics Subject Classification. Primary 58F11; Secondary 22D40, 28D20. 
by translations on any finite-volume homogeneous space $H / \Lambda$ of $H$. Rigidity properties of these $G$-actions and their restrictions to lattice subgroups follow from the theorem. We could state similar results for actions of semisimple groups (and their lattice subgroups), but here we have opted to avoid the technical details this would involve.

COROLlaRY 1. Suppose $G, H_{1}, H_{2}$ are connected noncompact simple Lie groups with trivial center, and let $\Lambda_{\imath}$ be a lattice in $H_{i}$. Embed $G$ in $H_{1}$ and $H_{2}$. Then any measure-theoretic isomorphism from the $G$-action on $H_{1} / \Lambda_{1}$ to the $G$-action on $\mathrm{H}_{2} / \Lambda_{2}$ is an affine map (a.e.).

For any lattice $\Gamma$ in $G$, we obtain a class of ergodic $\Gamma$-actions by restricting these natural $G$-actions. The $\Gamma$-actions are rigid because their inductions are rigid $G$-actions. More precisely:

COROllary 2. Suppose $G, H_{\imath}, \Lambda_{i}$ are as in Corollary 1 , and let $\Gamma$ be a lattice in $G$. Then any measure-theoretic isomorphism of the $\Gamma$-actions on $H_{1} / \Lambda_{1}$ and $H_{2} / \Lambda_{2}$ is affine (a.e.).

Though little is known about the possible actions of $G$ on a manifold, the Theorem implies that the natural actions of $G$ on a homogeneous space $H / \Lambda$ can be picked out by the action of a single unipotent element:

COROLLARY 3. Let $G$ and $H$ be connected noncompact simple Lie groups with finite center, and let $\Lambda$ be a lattice in $H$. Assume $\mathbf{R}$-rank $G \geq 2$. Suppose $G$ acts measurably on $H / \Lambda$ in an arbitrary way that preserves the finite measure. If some unipotent element of $G$ acts by an ergodic translation of $H / \Lambda$, then all elements of $G$ act by translations (a.e.).

Now let us consider only properly ergodic free actions with finite invariant measure. In this situation Dye [1] has proved that all actions of amenable discrete groups are orbit equivalent. At the other extreme, Zimmer's rigidity theorem [5] implies, under appropriate hypotheses, that actions of different connected noncompact simple Lie groups are never orbit equivalent. Zimmer's theorem has strong implications for the rigidity of actions of lattice groups, but it is possible for nonisomorphic lattices to have actions that are orbit equivalent. For a certain natural class of $\Gamma$-actions, however, we can show such a phenomenon does not occur:

COROLLARY 4. Let $\Gamma$ and $\Lambda$ be lattices in connected noncompact simple Lie groups $G$ and $H$ with trivial center. Assume $\mathbf{R}$-rank $G \geq 2$. Suppose $\Gamma$ and $\Lambda$ act ergodically by isometries of compact connected Riemannian manifolds $M$ and $N$. If the $\Gamma$-action on $M$ is orbit equivalent to the $\Lambda$-action on $N$, then $\Gamma \cong \Lambda$ and, identifying $\Gamma$ with $\Lambda$ under this isomorphism, the $\Gamma$-actions on $M$ and $N$ are isomorphic.

\section{REFERENCES}

1. H. Dye, On groups of measure preserving transformations. I, II, Amer. J. Math. 81 (1959), 119 159; ibid. 85 (1963), 551-576.

2. Donald Ornstein and Benjamin Weiss, Geodesic flows are Bernoulli, Israel J. Math. 14 (1973), 184- 198. 
3. Marina Ratner, Rigidity of horocycle flows, Ann. of Math. (2) 115 (1982), 597-614.

4. Dave Witte, Rigidity of some translations on homogeneous spaces, preprint.

5. Robert J. Zimmer, Strong rigidity for ergodic actions of semisimple Lie groups, Ann. of Math. (2) 112 (1980), 511-529.

Department of MAThematics, University of Chicago, Chicago, Illinois 60637 